\title{
Non-traditional Machining Processes in Bio-medical Applications - A Review
}

\author{
Mohammed Shahabaz Khan ${ }^{1}$, Mohammed Zafar ${ }^{1}$, Jeevan T. P.,** \\ ${ }^{1}$ U.G. Student, Department of Mechanical Engineering, Malnad College of Engineering, Hassan, Karnataka, India \\ ${ }^{2}$ Assistant Professor, Department of Mechanical Engineering, Malnad College of Engineering, Hassan, Karnataka, India
}

Copyright $@ 2017$ by authors, all rights reserved. Authors agree that this article remains permanently open access under the terms of the Creative Commons Attribution License 4.0 International License

\begin{abstract}
The main purpose of this paper is to review recent developments and research issues of non-traditional machining processes (NTM) in bio-medical engineering. The miniaturization ability has enabled NTM processes to be applied in many areas of biology, medicine, and biomedical engineering. As these processes have been proved much more reliable, economic and accurate in machining materials used in the above fields as these processes have set new standards of how easily a tough and resilient material can be machined with such precision and in turn lead to saving lives of millions of human beings who come under such surgical circumstances. This paper highlights the machining processes involved in the making of tools and equipment used in various Bio-medical fields like dentistry, optometry, implant tools and much more.
\end{abstract}

Keywords Non-traditional Machining, Bio-medical Engineering, Miniaturization

\section{Introduction}

In the past two decades, healthcare expenditure has grown rapidly. Solving the challenge of the increasing costs of healthcare requires a multidisciplinary approach. Manufacturing is a critical part of the solution. Manufacturing in the medical market demands superior accuracy, requires top precision, and needs the flexibility to accommodate the incredible demands of a constantly-changing and rapidly evolving industry. Since the 1940s, a revolution in manufacturing has been taking place that once again allowed manufacturers to meet the demands imposed by increasingly sophisticated designs and durability; but in many cases they were nearly difficult to machine materials. This manufacturing revolution is now, as it has been in the past, centered on the use of new tools and new forms of energy. This has resulted in the introduction of new manufacturing processes used for material removal known today as NTM Processes.
Machining is widely utilized in health care and is critical to the efficacy and outcome of the procedure. For example, needle insertion for vessel access, drug delivery, or biopsy is a tissue (Tissue is the workpiece in biomedical machining, the work material ranges widely; from soft tissue to hard bone and tooth) cutting and biomedical machining process. Dental drilling and root canal require extensive milling, grinding, and drilling processes [1]. Various NTM process specializes in manufacturing medical devices, medical components, medical tools and implants for all aspects of the field including diagnosis, therapy and surgery.

NTM is an advanced type of machining which is employed to machine extremely hard and brittle materials into intricate and complicated shapes by using various sources of energies like mechanical, chemical, thermal, electrical or a combination of these energies.

According to recent developments in these processes, some of the processes like micromachining, wire electrical discharge machining (WEDM), Water jet machining (WJM), ultrasonic machining (USM), laser beam machining (LBM) etc. have found extensive applications in the field of medicine and machining of medical equipments. In the course of the last decades medical surgery has been developing rapidly. A significant qualitative change is being noted at present when the development of technical and biological sciences is moving the field of medical surgery forward [2, 3]. In the present review an attempt has been made to throw light on some of the case studies and reviews in this regard.

\section{Why NTM Process in Current Scenario?}

Manufacturing industry is becoming ever more time and quality conscious with regard to the global competence, and the need to use complicated and precise components having some special shape requirements. The demand for materials made from exotic, high strength and temperature resistive materials, tool and die steels and advanced 
materials are growing day by day. These trends have placed a premium on the use of new and advanced technologies for quickly turning raw materials into usable goods; with less time or possibly no time being required for tooling. The conventional machining processes, in spite of recent technical advancement, are inadequate to machine complex shapes in hard, high strength temperature resistant alloys and die steels. Keeping these requirements into mind, a number of NTM processes have been developed that remove excess materials by various techniques involving mechanical, thermal ,electrical, chemical or combinations of these energies but do not use a sharp cutting tools as it needs to be used for traditional manufacturing processes. Nontraditional processes are sometimes applied to increase productivity either by reducing the number of overall manufacturing operations required to produce a product or by performing operations faster than the previously used method. It is also used to reduce the number of rejects by increasing repeatability, reducing in - process breakage of fragile work pieces, or by minimizing detrimental effects on work pieces, or by minimizing detrimental effects on work piece properties.

\section{Non-traditional Machining Processes in Bio-medical Applications}

The current state of the art in the sphere of utilization of NTM techniques of cutting and machining in medical applications and related issues are discussed with respect to different NTM techniques like micromachining, wire electric discharge machining (WEDM), water jet machining, laser beam machining and ultrasonic machining

\subsection{Micromachining}

Micromachining technology increasingly plays a decisive role in the miniaturization of components ranging from biomedical applications to chemical micro-reactors and sensors.

\subsubsection{Micromachined Needles}

Micromachining enables fabrication of needles smaller than $300 \mu \mathrm{m}$, which is the limit of conventional machining methods. Typically, the length of the Micromachined needles is less than $1 \mathrm{~mm}$. Microneedles have been used for drug delivery, bio-signal recording electrodes, blood extraction, fluid sampling, cancer therapy, and microdialysis. Frequently, microneedles are integrated and used in conjunction with microfluidic systems. Solid and hollow microneedles have been fabricated out of silicon, glass, metals, and polymers using micromachining processes. Microneedles have been demonstrated with various body shapes (cylindrical, canonical, pyramid, candle, spike, spear, square, pentagonal, hexagonal, octagonal and rocket shape) and tip shapes (volcano, snake fang, cylindrical, canonical, micro-hypodermis and tapered).

Figure 1 shows solid microneedles fabricated by reactive ion etching of silicon [4] and hollow microneedles fabricated by laser machining of a polymer.

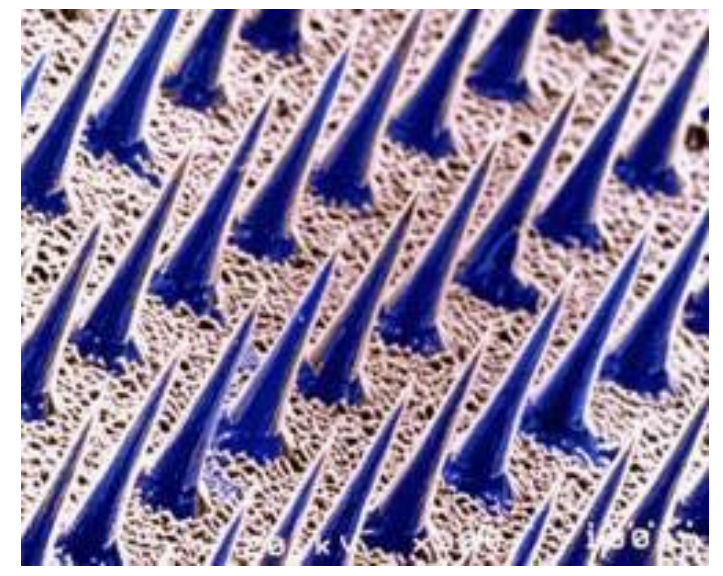

Figure 1. Micromachined needles: silicon based solid needles

\subsubsection{Micromachined Silicon Ultrasonic Surgical Tool for Eye Surgery}

Micromachined silicon ultrasonic surgical tools for phacoemulsification have been developed (Figure 2). In eye surgery there are many times when precision cutting is required. Highly sharpened steel, ceramic, or diamond scalpel blades are used, but are expensive to produce. Disposable silicon micromachined scalpels are an attractive alternative. They can be batch fabricated and sharpened to an atomic edge along their crystal planes. They are already being used at the Fyodorov Eye Care Center, Moscow, Russia, in nonpenetrating deep sclerectomy operations for the treatment of glaucoma [5].

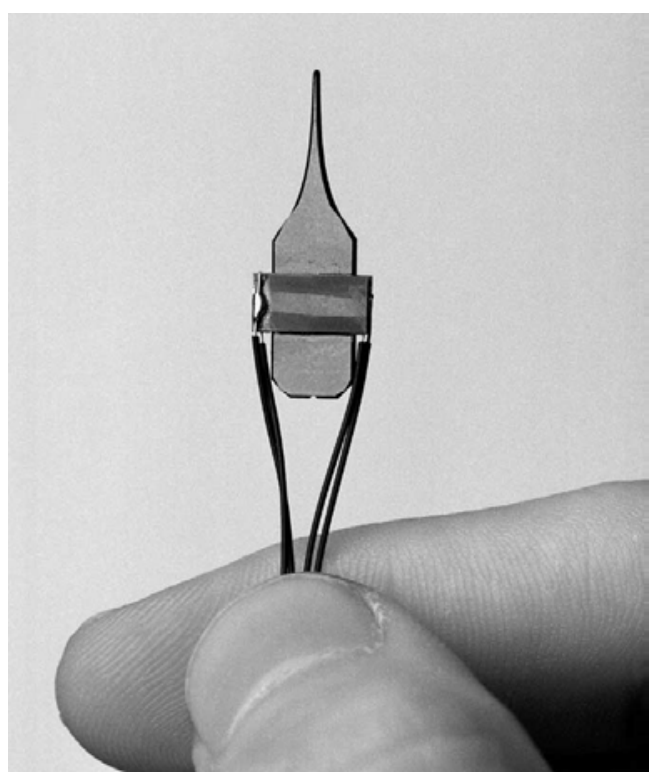

Figure 2. Ultrasonic phacoemulsification tool (Courtesy of J. Miller, University of Wisconsin, Madison) 


\subsubsection{Micromachined Pressure Sensors}

The first Micromachined devices to be used in the biomedical industry were reusable blood pressure sensors in the 1980s. Micromachined pressure sensors have the largest class of applications including disposable blood pressure, intraocular pressure (IOP), intracranial pressure (ICP), intrauterine pressure, and angioplasty [6].

\subsection{Wire Electric Discharge Machining (WEDM)}

Wire electrical discharge machining (WEDM) is extensively used in machining of conductive materials when precision is of prime importance.

\subsubsection{Machining of a Surgical Self-locking Bone Screw by WEDM}

The necessity of machining a screw which locks itself came in many medical cases one such being the case of slipped capital femoral epiphysis (SCFE) in which children are born with a critical case where the ball of the hip joint is separated from the thigh bone. Here, a screw needs to be induced which should lock itself so that the newly born may not have to face much pain. Earlier stainless steel screws were used but now a day's titanium screws are used. WEDM [7-9] is used in the machining of such screws where the machine makes a series of wire-cuts radially, down the side and length of the screw, $120^{\circ}$ apart. In use, the screw is threaded into a pre-drilled, "tap-size" hole in the bone with a hex key. The slot configuration lets a portion of the screw body flex inward when threaded under pressure. The compressed screw sections remain in tension and press outward like an expanding collet or mandrel, preventing the screw from loosening or moving.

\subsubsection{Machining of Self-retaining Suture Anchor by WEDM.}

When there was a dislocation in the bone-tendon regions, old conventionally machined screws were inserted to fix the same and it were very difficult to insert and the suture anchors had to be inserted in a pre-drilled hole and had to be held in place until the threads of the screw engage with the sides of the pre-drilled hole. With this invention, using the WEDM process, it was possible to machine an anchor which was self-retaining and was able to fix tendons and ligaments to bone with less difficulty when compared to the previously occurring anchors [10].

\subsection{Water Jet Machining}

Using waterjets instead of rigid drill bits for bone drilling can be beneficial due to the absence of thermal damage and a consequent sharp cut. Additionally, waterjet technology allows the development of flexible instruments that facilitate maneuvering through complex joint spaces [11].

\subsubsection{Surgical Cutting and Ablation by Water Jet Machining [12]}

Water jet cutting is a non-thermal technology for cutting of tissues, including bone [13, 14]. Advances in water jet systems are facilitating their increased use in new medical ablation or cutting applications in fields as diverse as cardiology, orthopaedics, ophthalmology, dermatology, oncology and neurosurgery. The key element is water jet travelling at high velocity. When the water stream strikes the tissue or bone, material can be rapidly removed by the erosive force of the water. The water jet method offers the advantages of no mechanical contact between tool and bone or tissue, with minimal mechanical force applied, minimal localized heat-affected zone, and accuracy of control. It should be noted that in established applications such as in cutting of steel and titanium by water-jet small diameter particles usually ground garent are injected into the water jet by means of a special nozzle. The water jet accelerates the particles which gather a large kinetic energy. Material removal is achieved by the combined effects of the particles and water jet. When softer biomaterials such as tissue are to be cut no such abrasive particles can be introduced, and cutting has to be effected solely by water. In the latter case pure water is replaced by saline.

\subsubsection{Disintegration of Bone Cement by Continuous and Pulsating Water Jet [15]}

Number of cemented and non-cemented endoprostheses reimplantations is increasing. Effective surgical process of total hip and knee replacement depends on technology. Problematic issues of artificial joints are wear and corrosion, which leads to aseptic failure of endoprostheses. The bio-material disintegration during revision arthroplasty issue is currently an existing problem of more surgeons even in regard to the fact that the bone tissue is a compact and living material. To make the damaged total cemented endoprosthesis extractable the interface between the bone and bone cement is inevitable to be disturbed .Negative side effects of using classical tools during reimplantation of cemented endoprostheses are distinctive heat and deformation impacts, loosing of biological potential. It is the application of the water jet cutting that offers eventuality of eliminating the negative features of classical tools and unconventional processes. Water jet cutting is also used in Endoprosthesis revision surgery - removing prostheses rapidly with little damage to the surrounding tissue [16].

\subsubsection{Pure Waterjet Drilling of Articular Bone}

The clinical application of waterjet technology for machining tough human tissues, such as articular bone, has advantages, as it produces clean sharp cuts without tissue heating. Additionally, water supply is possible via flexible tubing, which enables minimally invasive surgical access. This pilot study investigates whether drilling bony tissue 
with pure waterjets is feasible. Water pressures between 20 and $120 \mathrm{MPa}$ with an orifice of $0.6 \mathrm{~mm}$ were used to create waterjets to drill blind borings in the talar articular surface of cadaveric calcaneus bones [17]. Figure 3 shows water jet drilled holes in femur bone.

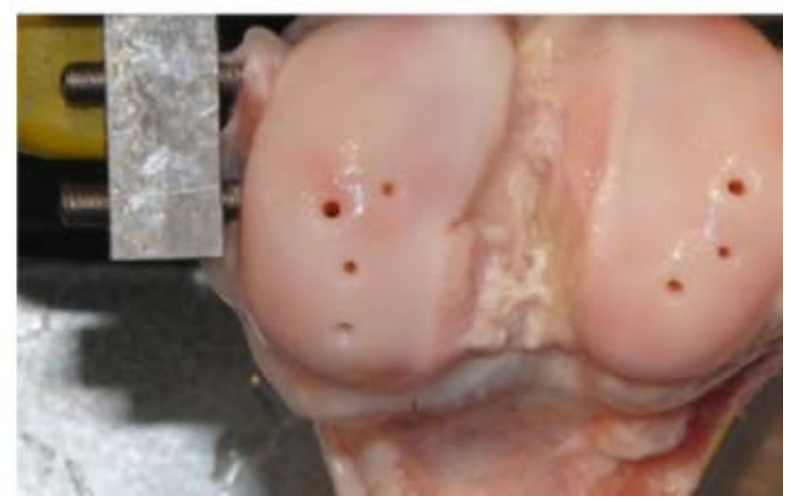

Figure 3. Water jet drilled holes in femur bone

\subsection{Ultrasonic Machining}

\subsubsection{Use of Ultrasonically-assisted Machining in Orthopaedic Surgeries}

Loughborough University researchers have developed a device which could revolutionize the way cutting, drilling and milling is done in manufacturing. The tool makes working on difficult-to-cut materials like aerospace-grade composites so easy it is like 'cutting through butter'. It involves a technique called ultrasonically-assisted machining (UAM), which uses a specially designed piezoelectric transducer working in tandem with a traditional turning, drilling or milling machine. The technique is currently being extended into biomedical applications such as drilling holes in bones for orthopaedic surgery $[18,19]$.

USM is also used to correct conditions of the jaw and face, to achieve a correct bite, an aesthetic face and an enlarged airway, sinus lift, alveolar ridge expansion, exposure of impacted canines, lateralization of the inferior alveolar nerve removal of osseous tissue close to the IAN, orthognathic surgery, autologous bone graft, harvesting, periodontal surgery, IAN transposition, alveolar distraction osteogenesis, and the removal of osseointegrated implants [20-23].

\subsubsection{Ultrasonic Machining for Bone and Hard Tissue [24]}

Ultrasonic techniques have been applied in case of machining of hard tissues as early as 1950s. Many efforts have been made in the field of dentistry. The initial study shows that ultrasonic cutting when compared to traditional burr produces superficial cuts which can be advantageous in controlling tissue damage.

\subsection{Laser Beam Machining}

Laser beam machining (LBM) is one of the most widely used thermal energy based non-contact type advance machining process which can be applied for almost whole range of materials.

From surgical instruments used in cutting and biopsy, to needles containing unusual tips and side wall openings, and puzzle chain linkages for flexible endoscopes, laser cutting provides higher precision, quality, and speed than traditional cutting techniques. Laser beam machining is used for Rotational Acetabular Osteotomy (RAO), to understand changes induced in bone in terms of temperature rise and thermal damage, feasibility of performing complete osteotomy, examine bone healing under functional loading [25-28].

Laser micromachining of silicon was also used to develop groove-like patterns that may be used to facilitate the orientation of human aortic vascular smooth muscle cells [29].

Laser surface texturing can be used to produce well defined micro-grooves on biomedical materials such as Ti-6Al-4V. Such micro-grooves can be optimized to improve the integration with surrounding tissue [30].

\subsection{Other NTM Processes}

In surgery, surgeons need to cut tissue and coagulate blood using heat to form clots for hemostasis. In the past, a heated, sharp blade was used to cut and coagulate tissue at the same time in surgery. This procedure is performed by electrosurgical devices today. The first electrosurgical device was developed by Harvey Cushing, a surgeon, and William Bovie, a physicist, in 1926. The fundamental principles used in electrosurgery are similar to EDM and resistance heating [1].

Abrasive machining has been applied not only to the manufacturing of biomedical devices but also to the direct patient treatment and care: Abrasive tissue removal, such as rotational atherectomy, skin treatment, and cutting of enamel; Machining of medical parts, such as surface conditioning of medical instrument blades, machining of medical ceramic parts, polishing of surface of artificial joints Dental hard tissue machining [31].

Cryogenic cooling is known to provide a very sustainable machining process because of its environmentally benign, and economically and societally-beneficial nature. In the context of biomedical machining, a key benefit of cryogenic machining [32] is the elimination of secondary cleaning processes usually necessitated to wash off contamination from flood coolant (water/oil emulsion).

\section{Conclusions}

Over the past five decades, more than twenty NTM processes have been invented and successfully implemented in production. The reason for the large 
number of NTM processes are that each process has its own characteristics, capabilities and limitations, so no process is best for all manufacturing situations.

Medical advances in the last decade have produced numerous surgical tools, drug delivery equipment, diagnostic devices, and surgically implantable devices with the aid of NTM processes. This proliferation created a major challenge to the high-volume producers of such ultra-precise devices.

NTM processes are powerful tools for enabling the miniaturization of devices useful in biomedical engineering. The need to precisely control gas and fluid flow is critical for diagnostic, surgical, and biomedical systems. With this as motivation, there have been many efforts to develop viable reliable low-cost high-precision micro machined surgical tools. NTM processes has the potential to reduce the cost of health-care management thus enables remote or small-scale clinics to offer fast high-quality tests.

\section{REFERENCES}

[1] Liang S.Y., Shih A.J. Biomedical Machining. In: Analysis of Machining and Machine Tools. Springer, Boston, MA, 2016.

[2] Ritter MA, Campbell ED. The survival of the cemented femoral component of a total hip replacement. Clin. Orthop. 243, pp. 143-147, 1989.

[3] Salgado AJ, Coutinho OP, Reis LR. Bone tissue Engineering: State of the Art and Future Trends. Macromol. Biosci. 2004, 4, pp. 743-765, 2004.

[4] Hospital, Sutton, Surrey.S. Henry, D. V. Mc Allister, M. G. Allen and M. R. Prausnitz, "Microfabricated Microneedles: A Novel Approach to Transdermal Drug Delivery,” Journal of Pharmaceutical Sciences, 87, pp. 922-925, 1998.

[5] T. V. Kozlova, N. F. Shaposhnikova, V. B. Scobeleva, and T. V. Sokolovskaya, "Non penetrating deep sclerectomy: Evolution of the method and prospects for development (review),” Ophthalmosurgery, vol. 3, pp. 39-54, 2000.

[6] Dr. Ramesh Ramadoss, MEMS devices for biomedical applications, electroiq.com/blog/2013.

[7] Holmes and Andrew, Machining of a surgical self-locking Bone Screw. Machining Interview, 2008.

[8] Lehmann, Charles Journal of Pediatric Orthopedics, 26(3), pp. 286-290, 2006.

[9] Ilchmann, T., Parsch, K. "Complications at screw removal in slipped capital femoral epiphysis treated by cannulated titanium screws." Archives of Orthopaedic and Trauma Surgery, 126, pp. 359-363, 2006.

[10] Inventor: Richard J. Deslauriers, 78 Joseph St., Waterbury, Conn. 06705, US Patent No. US006149653A, 2000.

[11] den Dunnen, S., Mulder, L., Kerkhoffs, G. M., Dankelman,
J., \& Tuijthof, G. J. Waterjet drilling in porcine bone: The effect of the nozzle diameter and bone architecture on the hole dimensions. Journal of the mechanical behavior of biomedical materials, 27, pp. 84-93. 2013.

[12] McGuinness, G. Surgical Cutting and Ablation by Energy Based Devices: Principles and Applications. International Conference on Advanced Manufacturing Systems and Technology, Mali Losinj, Croatia, 16-17 June, 2011.

[13] Tschan, C.A., Tschan, K., Krauss. J.K., Oertel, J. First experimental results with a new waterjet ssector: Erbejet, Acta Neurochirurgica, 151, pp. 1473-1482, 2009.

[14] Spahn, G., Froeber, R., Lin W., Treatment of chondral defects by hydro jet. Results of a preliminary scanning electron microscopic evaluation, Archives of Orthopaedic and Trauma Surgery, 126: pp. 223-227, 2006.

[15] Hloch, S., Kl’oc, J., Hreha, P., Magurová, D., Kozak, D. and Knapčíková, L., Water jet technology using in orthopaedic surgery. hip, 3500(4000), pp. 4500, 2013.

[16] Honl, M., R. Rentzsch, G. Müller, C. Brandt, A. Bluhm, E. Hille, H. Louis, M. Morlock. The use of Water-Jetting Technology in Prostheses Revision Surgery - First Results of Parameter Studies on Bone and Bone Cement, Journal of Biomedical Materials Research Part B: Applied Biomaterials, vol. 53 (6), pp. 781- 790, 2000.

[17] Steven den Dunnen, Gert Kraaij, Christian Biskup, Gino M.M.J. Kerkhoffs, Gabrielle J.M. Tuijthof, Pure Waterjet Drilling of Articular Bone: An in vitro Feasibility Study Journal of Mechanical Engineering 59, vol. 7-8, pp. 425-432, 2013.

[18] Alam, K., Mitrofanov, A.V. And Silberschmidt, V.V. Experimental investigations of forces and torque in conventional and ultrasonically-assisted drilling of cortical bone. Medical Engineering and Physics, 33 (2), pp. 234-239, 2010.

[19] Khurshid Alam, Edris Hassan \& Issam Bahadur. Experimental measurements of temperatures in ultrasonically assisted drilling of cortical bone, Biotechnology \& Biotechnological Equipment, 29:4, pp. 753-757, 2015.

[20] Giraud JY. Bone cutting. Clin. Physics and Physiological Measurement, 1991.

[21] Steiger RN, Pandey R, McLardy-Smith P. Ultrasonically driven tools. J Arthroplasty; 11: pp. 120-121, 1996.

[22] Caillouette JT, Gorab RS, Klapper RC, Anzel SH. Revision arthroplasty facilitated by ultrasonic tool cement removal. Part I: In vitro evaluation. Orthop Rev; pp. 353-357, 1991.

[23] Klapper RC, Caillouette JT, Callaghan JJ, Hozack WJ. Ultrasonic technology in revision joint arthroplasty. Clin Orthop, pp. 147-154, 1992.

[24] Dahotre, Narendra B., and Sameehan Joshi. Machining of bone and hard tissues. Springer, 2016.

[25] Kim D, H. Owada, N. Hata, T. Dohi. An Er: YAG Laser Bone Cutting Manipulator for Precise Rotational Acetabular Osteotomy, Conf. Proc. IEEE Engineering in Medicine and Biology Society, vol.4., 2004. 
[26] Eyrich, G. K. H. Laser-Osteotomy Induced Changes in Bone, Medical Laser Application, vol. 20(1), pp. 25-36, 2005.

[27] Zhang, X., S. Xie, Z. Zhan, Q. Ye. One Dimension Dynamic Ablation of Bovine Shank Bone with Pulse CO2 Laser, Progress in Biomedica Optics and Imaging, vol. 8 (49), pp. 68260I.1- 68260I.8, 2007.

[28] Kuttenberger, J.J., S. Stübinger, A. Waibel, M.Werner, M. Ivanenko, P. Hering, B.von Rechenberg, R. Sader, H. F. Zeilhofer. Computer-guided $\mathrm{CO}_{2}$-laser Osteotomy of the Sheep Tibia: Technical Prerequisites and First Results, Photomedicine and Laser Surgery, vol. 26 (2), pp. 129-136, 2008.

[29] Miller, Philip R., et al. "Laser micromachining for biomedical applications." Journal of the Minerals, Metals and Materials Society 61.9, pp. 35-40, 2009.

[30] Fasasi, A. Y., Mwenifumbo, S., Rahbar, N., Chen, J., Li, M., Beye, A. C., ... \& Soboyejo, W. O. (2009). Nano-second UV laser processed micro-grooves on Ti6Al4V for biomedical applications. Materials Science and Engineering: C, 29(1), 5-13.

[31] Inderscience Publications Retrieved November 25, 2017, from http://www.inderscience.com/info/ingeneral

[32] Jawahir, I. S., D. A. Puleo, and J. Schoop. "Cryogenic machining of biomedical implant materials for improved functional performance, life and sustainability." Procedia CIRP46 (2016): 7-14. 